\title{
Identification of novel endogenous antisense transcripts by DNA microarray analysis targeting complementary strand of annotated
} genes

\author{
Koji Numata ${ }^{1}$, Yuko Osada ${ }^{2}$, Yuki Okada ${ }^{2}$, Rintaro Saito ${ }^{2,3}$, Noriko Hiraiwa ${ }^{4}$, \\ Hajime Nakaoka ${ }^{5}$, Naoyuki Yamamoto ${ }^{5}$, Kazufumi Watanabe ${ }^{6}$, \\ Kazue Okubo $^{7}$, Chihiro Kohama ${ }^{8}$, Akio Kanai ${ }^{2,3}$, Kuniya Abe ${ }^{1,8}$ and \\ Hidenori Kiyosawa*1,8,9
}

Address: ${ }^{1}$ Technology and Development Team for Mammalian Cellular Dynamics, BioResource Center (BRC), RIKEN Tsukuba Institute, Ibaraki 305-0074, Japan, ${ }^{2}$ Institute for Advanced Biosciences, Keio University, Yamagata 997-0017, Japan, ${ }^{3}$ Department of Environmental Information, Keio University, Fujisawa 252-8520, Japan, ${ }^{4}$ Experimental Animal Division, BioResource Center (BRC), RIKEN Tsukuba Institute, Ibaraki 3050074, Japan, ${ }^{5}$ C's Lab Co Ltd Maruito Sapporo Bldg, 7F Kita 2 Nishi 1, Kita-ku, Sapporo 060-0002, Japan, ${ }^{6}$ Custom Biotechnology Service Group, Hokkaido System Science Co Ltd, 2-1 Shinkawa Nishi 2-1, Kita-ku, Sapporo 001-0932, Japan, ${ }^{7}$ Genostaff Inc., Kawauchi Bldg 6F 1-4-4, Nezu, Bunkyo-Ku, Tokyo 113-0031, Japan, ${ }^{8}$ Graduate School of Life and Environmental Sciences, University of Tsukuba, Ibaraki 305-0006, Japan and ${ }^{9}$ Technology and Development Team for BioSignal Program, BioResource Center (BRC), RIKEN Tsukuba Institute, Ibaraki 305-0074, Japan

Email: Koji Numata - koji.numata@gmail.com; Yuko Osada - yuko.osada@gmail.com; Yuki Okada - okayan@gmail.com;

Rintaro Saito - rsaito@sfc.keio.ac.jp; Noriko Hiraiwa - hiraiwa@rtc.riken.jp; Hajime Nakaoka - nakaoka@cslab.co.jp;

Naoyuki Yamamoto - yamamoto@cslab.co.jp; Kazufumi Watanabe - watanabe@hssnet.co.jp; Kazue Okubo - okuboka@genostaff.com;

Chihiro Kohama -s0921075@u.tsukuba.ac.jp; Akio Kanai - akio@sfc.keio.ac.jp; Kuniya Abe - abe@rtc.riken.jp;

Hidenori Kiyosawa* - kiyosawa@rtc.riken.jp

* Corresponding author

Published: 22 August 2009

BMC Genomics 2009, 10:392 doi:10.1186/147|-2164-10-392
Received: 27 February 2009

Accepted: 22 August 2009

This article is available from: http://www.biomedcentral.com/I47I-2/64/I0/392

(c) 2009 Numata et al; licensee BioMed Central Ltd.

This is an Open Access article distributed under the terms of the Creative Commons Attribution License (http://creativecommons.org/licenses/by/2.0), which permits unrestricted use, distribution, and reproduction in any medium, provided the original work is properly cited.

\begin{abstract}
Background: Recent transcriptomic analyses in mammals have uncovered the widespread occurrence of endogenous antisense transcripts, termed natural antisense transcripts (NATs). NATs are transcribed from the opposite strand of the gene locus and are thought to control sense gene expression, but the mechanism of such regulation is as yet unknown. Although several thousand potential sense-antisense pairs have been identified in mammals, examples of functionally characterized NATs remain limited. To identify NAT candidates suitable for further functional analyses, we performed DNA microarray-based NAT screening using mouse adult normal tissues and mammary tumors to target not only the sense orientation but also the complementary strand of the annotated genes.
\end{abstract}

Results: First, we designed microarray probes to target the complementary strand of genes for which an antisense counterpart had been identified only in human public cDNA sources, but not in the mouse. We observed a prominent expression signal from $66.1 \%$ of 635 target genes, and 58 genes of these showed tissue-specific expression. Expression analyses of selected examples (Acaa lb and Aard) confirmed their dynamic transcription in vivo. Although interspecies conservation of NAT expression was previously investigated by the presence of CDNA sources in both species, our results suggest that there are more examples of human-mouse conserved NATs that could not be identified by cDNA sources. We also designed probes to target the complementary strand of wellcharacterized genes, including oncogenes, and compared the expression of these genes between 
mammary cancerous tissues and non-pathological tissues. We found that antisense expression of 95 genes of 404 well-annotated genes was markedly altered in tumor tissue compared with that in normal tissue and that 19 of these genes also exhibited changes in sense gene expression. These results highlight the importance of NAT expression in the regulation of cellular events and in pathological conditions.

Conclusion: Our microarray platform targeting the complementary strand of annotated genes successfully identified novel NATs that could not be identified by publically available cDNA data, and as such could not be detected by the usual "sense-targeting" microarray approach. Differentially expressed NATs monitored by this platform may provide candidates for investigations of gene function. An advantage of our microarray platform is that it can be applied to any genes and target samples of interest.

\section{Background}

There is a growing body of evidence that natural antisense transcripts (NATs) play important regulatory roles in various biological processes. NATs are usually transcribed from the opposite strand of a particular gene locus, and they are thought to regulate sense gene expression $[1,2]$. One of the proposed models of NAT-mediated regulation is for the antisense transcript to act as a cis-repressor of gene expression from the sense strand. For example, in early embryogenesis, transcription of the antisense genes Tsix and Air determines the fate of expression of their sense partners Xist and Igf2r, respectively [3,4]. The appearance of NATs within several imprinted loci suggests that NATs may regulate gene expression by controlling the epigenetic status of surrounding genes [5-7]. Moreover, NATs may function in pathological conditions by causing epigenetic alterations such as histone modification and DNA methylation $[8,9]$.

The other primary model of NAT-mediated gene regulation is induction of the production of small RNAs from NAT loci and their subsequent function in RNA interference (RNAi) pathways. Endogenous small interfering RNA (endo-siRNA) molecules, generated from NAT loci, are induced specifically under conditions of salt stress and immune response in plants [10-15]. Recent experimental data also suggests the presence of NAT-associated endosiRNA molecules in animals [16-18].

Although the number of NATs thought to have biological functions has gradually increased, the functions of most NATs discovered in recent large-scale in silico studies are unknown. Computational identification of NATs is based mostly on the analysis of cDNA and EST sequence collections by sequence alignment, and this process has identified several thousand sense-antisense pairs [19]. However, in principle, cDNA sequencing accumulates data on transcripts with poly(A)-stretches and does not access the non-poly-adenylated population of transcripts. A recent genome-wide tiling array study of the human genome revealed that many genomic regions that could not be identified from cDNA collections are apparently transcribed and tend not to be poly-adenylated [20]. This finding indicates that antisense transcriptome analyses based solely on cDNA information may be inefficient. In addition, most publicly available cDNA sequences are derived from normal cellular conditions, such as normal adult tissues, and thus are not useful for the identification of NATs specific to abnormal cellular conditions.

To discover novel NATs expressed under various biological conditions, we proposed a microarray-based technique involving the use of 60-mer oligonucleotide DNA probes selected from the complementary sequences of cDNAs (i.e., known genes), referred to as artificial antisense sequence (AFAS) probes. This approach has the ability to detect antisense expression that cannot be identified by using information from the cDNA and EST collections and has the advantage of compatibility with the computational methodology widely used for sense gene expression analysis [21]. We performed microarray analyses with AFAS probes by using oligo-dT and random primed target samples to provide a comprehensive approach for the detection of novel non-poly-adenylated transcripts in the antisense transcriptome.

Here, we designed AFAS probes to correspond to the antisense strand of well-studied selected genes, including oncogenes and tumor suppressor genes, imprinted genes, and human-mouse orthologous genes. We studied the expression profiles of targeted transcripts in normal mouse adult tissues and in mouse mammary tumor virus (MMTV)-induced mammary tumors. This technique is applicable to all genes and sample types and can be used for antisense expression identification that is not possible by using conventional cDNA information alone.

\section{Results}

\section{AFAS probes detect previously known NATs}

To verify whether our methods can detect NAT expression, we initially examined the signal intensities of AFAS probes that targeted previously identified antisense transcripts. 
For example, AFAS probes designed for Tsix, which reflects the abundance of Xist RNA, detected expression in the 11 adult mouse tissues (mixed males and females), but not in the testis, as expected (see Additional file 1). Such expression patterns were detected only for probes corresponding to the exonic-overlapping regions between Tsix and Xist, and also for the sense probes corresponding to Xist RNA (see Additional file 1). This finding suggests that AFAS probes for Tsix can identify not only the presence of its antisense counterpart, but also its exonic regions. In addition, AFAS probes for several imprinted genes (Igf2r, Kcnq1, Gnas, Dio3, and Ube3a), which are known to give rise to antisense transcripts [5], also gave prominent signals (see Additional file 2). Moreover, previously known antisense transcripts, such as those arising from $M \gamma c$ (myelocytomatosis oncogene) [22] and Tgfb2 (transforming growth factor beta2) [23], were also detected by our microarray platform (data not shown). Although the number of documented NAT examples in normal mouse adult tissues is limited, the mean signal intensities generated by AFAS probes corresponding to these genes were higher than those for the negative control genes. The control genes comprise a set of randomly selected genes, with- out cDNA, EST, and CAGE tags in the antisense orientation (Figure $1 \mathrm{~A}, P=5.1 \mathrm{e}-12$ by Welch's t-test). These data indicate that endogenous NAT expression of known genes is potentially detectable by probes designed for the complementary strand of known genes.

\section{Global analyses of AFAS probes}

Before screening for novel NATs using AFAS probes, we first analyzed the global tendency of signal intensities from all AFAS probes applied to our custom microarray platform. Because Northern blot analyses for particular gene loci have previously shown that NATs tend to be poly(A)-negative [24], we checked whether our AFAS probes also showed this tendency in normal mouse tissue expression profiling. A significantly higher number of AFAS probes than sense probes detected transcripts only within random-primed samples, but not among the oligodT primed targets $(P<2.2 e-16$, Fisher's exact test, see Additional file 3 ). This result indicates that transcripts detected by AFAS probes also lack poly(A)-tails, similar to the finding for NATs characterized by Northern blot analyses [24]. Also, the number of sense probes detecting transcripts in both oligo-dT and random primed samples was
A

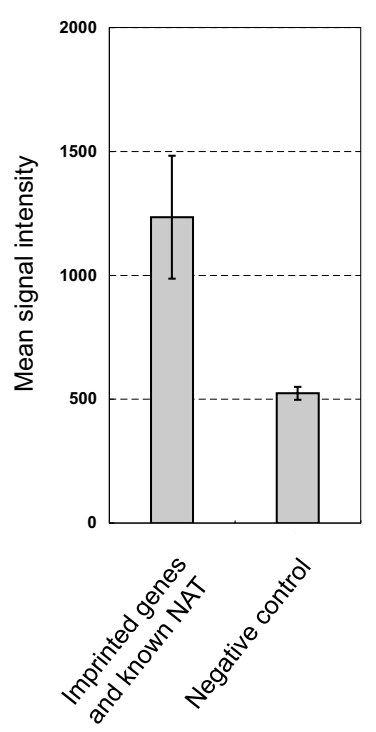

B

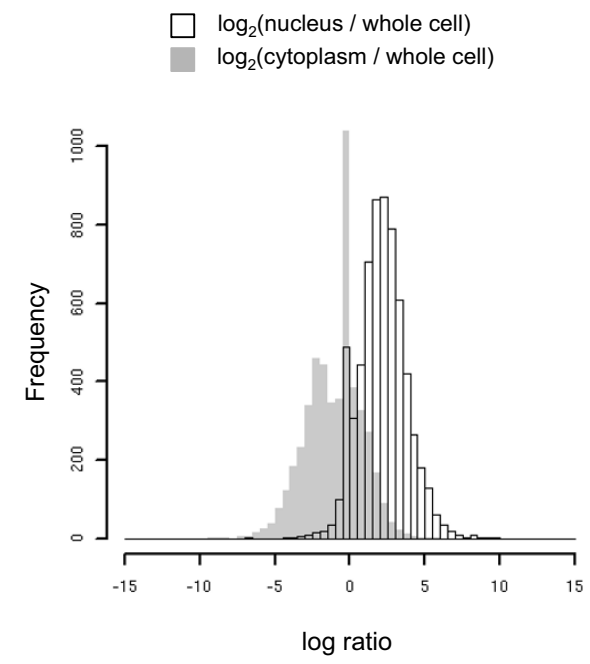

$\log _{2}$ (nucleus / whole cell)

$\log _{2}$ (cytoplasm / whole cell)

Relative position of mRNA

\section{Figure I}

Global tendency of AFAS probes in normal tissues. (A) Mean signal intensities of AFAS probes were compared between sets of known NAT-associated genes (including imprinted genes) and negative controls. Negative controls with no cDNA, EST, and CAGE tags in their antisense orientation were randomly picked from NCBI RefSeq. (B) Nuclear enrichment (grey bars) was measured by $\log _{2}\left(S_{\text {nucleus }} / S_{\text {whole-cell }}\right)$, where $S_{\text {nucleus }}$ and $S_{\text {whole-cell }}$ denote signals from nuclear fractions and from whole cells (NIH3T3), respectively. Cytoplasmic enrichment (open bars) was calculated by $\log _{2}\left(\mathrm{~S}_{\text {cytoplasm }} / \mathrm{S}_{\text {whole-cell }}\right.$ ), where $\mathrm{S}_{\text {cyto- }}$ plasm denotes signals from the cytoplasmic fraction. All signal intensities were obtained from the experiments by using randomprimed samples. (C) The sum of Z-scores for every relative position is indicated. For each tissue, the positional preference of NAT expression was measured by Z-scores calculated from the median signal values of every position. Expression data were obtained from random-primed samples. 
higher than that of antisense probes $(P<2.2 \mathrm{e}-16$, Fisher's exact test, see Additional file 3 ). This finding indicates that sense transcripts with poly(A)-tails can be identified by both priming methods, because sense probes target the protein-coding strand of the mRNA, which is expected to have a poly(A)-tail. Another characteristic of endogenous NATs is their nuclear localization [24]. A distribution comparison of AFAS probe signals between nuclear and cytoplasmic fractions clearly showed nuclear enrichment of detected transcripts (Figure $1 \mathrm{~B}, P<2.2 \mathrm{e}-16$, Median test).

Several large-scale studies using information in cDNA and EST collections and from genome-wide tiling arrays in yeast previously showed that NATs tend to be transcribed from the 3 ' region of its counterpart mRNA [25-27], thus implying the presence of regulatory mechanisms involving tail-to-tail overlapping. We also observed this characteristic for the AFAS probes, because the AFAS probe signals clearly showed positional preference relative to the sense mRNA (Figure 1C). This result indicates that AFAS probes indeed detect the positional bias of antisense transcription. Similarly, we also observed higher signals within 5' regions (Figure 1C), thus suggesting that NATs may also arise near the transcriptional start site, as previously shown for head-to-head overlapping NATs such as WT1, Sphk1, and Tsix [28-30].

\section{Novel conserved NAT detection by normal tissue profiling}

To test the ability of AFAS probes to detect novel NATs, we initially applied our microarray approach to the humanmouse orthologous gene set. In many studies, inter-species conservation of NATs is implied by the presence of common cDNA sequences between the two species [26,31,32]. However, recent genome-wide tiling array and CAGE analyses revealed that a large fraction of the genome is transcribed [33,34], indicating that current cDNA collection is not sufficient for comprehensive comparative genomics, including comparative antisense transcriptome analyses. In this situation, the use of AFAS probes corresponding to genes for which the antisense counterpart has been identified in humans, but not in mice, may lead to the detection of novel conserved NATs (Figure 2).

We designed AFAS probes corresponding to 635 mouse orthologous partners, for which the antisense counterpart has been identified in humans, but not in mice (one sense and one antisense probe were designed per gene). We then profiled the expression of these genes to detect antisense expression within 12 normal mouse tissues. We identified $420(66.1 \%)$ probes that gave a signal (signal intensity $\geq 100$, which is our empirically defined criterion), at least in a single particular tissue, and 58 of these (9.2\%) showed tissue-specific expression. Probes of 120 genes gave signals with a higher than average intensity according to inter-array normalization (see Additional file 4 ). These results suggest that many NATs identified only in the human cDNA collection may also be expressed in mice.

We attempted to validate the expression of two candidate conserved NATs (antisense of Acaa1b and Aard) by performing Northern and in situ hybridization (ISH) analyses. Whereas human ACAA1 (acetyl-Coenzyme A acyltransferase 1) overlaps with DLEC1 (deleted in lung and esophageal cancer 1) in a tail-to-tail overlapping manner, its orthologous counterpart in the mouse genome (Acaa1b and Dlec1) shows a tail-to-tail relationship but not a reciprocal overlapping relationship, according to the annotated gene structure (see Additional file 5). Both microarray and Northern analyses confirmed that the Acaa $1 b$ sense transcript is expressed within liver and kidney (see Additional file 5). Northern analyses were not able to detect the antisense transcript of $A c a a 1 b$ from either poly(A)+ or total RNA (data not shown), but quantitative RT-PCR, ISH and microarray analyses were able to detect this transcript within the testis and kidney (see Additional file 5). This result implies that NATs detected by microarray analysis using AFAS probes are transcribed in vivo.

We also analyzed the expression of Aard (alanine- and arginine-rich domain-containing protein), which is a functionally uncharacterized gene but is known to be expressed within the adult testis and XY fetal gonad [35]. In humans, exons of AARD (also known as C8orf85) overlap with that of an unnamed uterus EST (GenBank: AK093981), whereas mouse Aard has no EST arising from the antisense strand (Figure $3 \mathrm{~B}$ ). Northern analysis confirmed that expression of the sense transcript of Aard was testis-specific (Figure 3C); however, Northern analysis of the antisense transcript showed laddered hybridization patterns for total RNA, but not for poly(A)+ RNA isolated from all samples (Figure 3D). By comparison, both the sense and antisense transcripts (Aard-AS) were detected by ISH within a particular region of the seminiferous tubules (Figure 4A,B), thus confirming that the Aard-AS is also expressed in the testis. In addition, Aard-AS was most likely located within the nucleus, whereas Aard was located within the cytoplasm (Figure 4C,D). Because ISH shows that Aard-AS is expressed in a particular region of the seminiferous tubules, we checked our microarray data on fractionated testis samples that reflected the three steps of spermatogenesis (i.e., pachytene spermatocytes, round spermatids, and elongated spermatids). We found that Aard-AS was expressed within the early period of spermatogenesis, whereas the sense transcript appeared at a later phase (Figure 4E). This finding shows that sense and antisense transcripts of Aard are transcribed exclusively and in a mutually antagonistic fashion during spermatogenesis. In addition, Aard-AS expression was detected only in the 


\section{Conserved sense-antisense pairs by cDNA analysis \\ (antisense counterpart has been identified both in human and mouse)}

\author{
AFAS probe target \\ (antisense counterpart has been identified \\ in humans, not in mice)
}
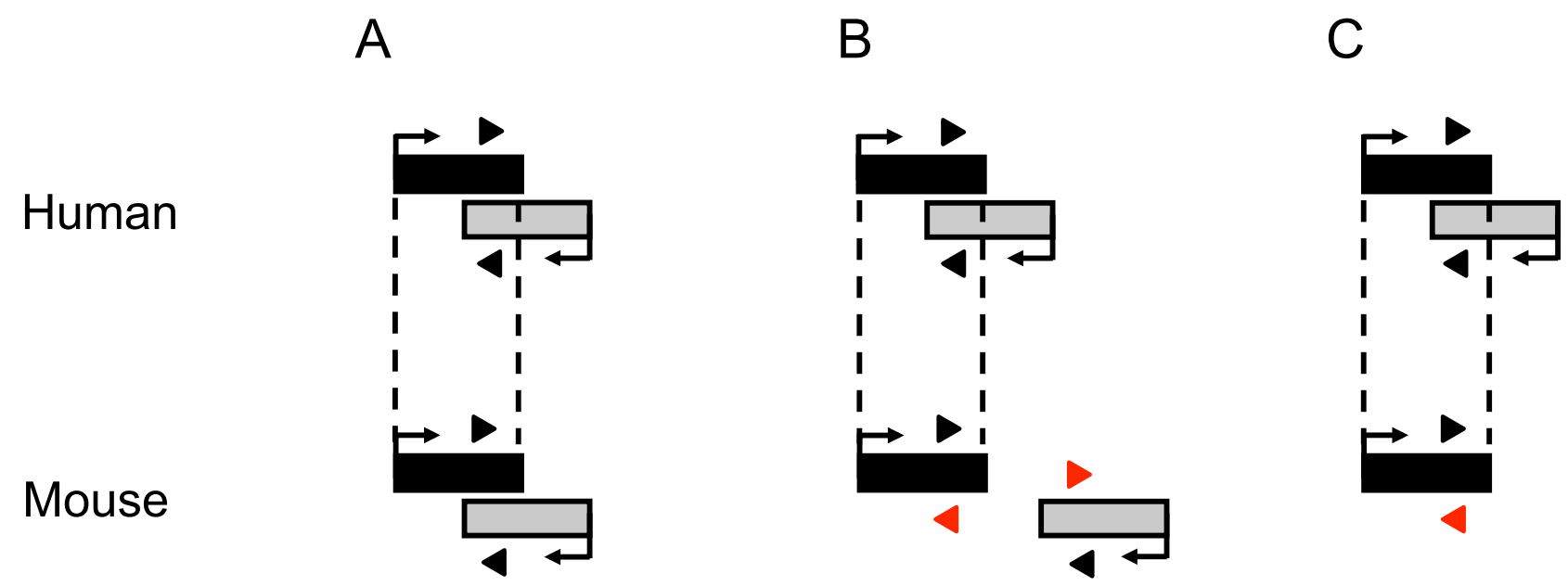

Figure 2

Schematic illustration of the strategy to discover novel conserved NATs. Conserved NATs were previously listed according to the presence of cDNA within humans and mice $(\mathbf{A})$. Black and grey boxes denote transcribed region identified by cDNA on plus and minus strand of the genome, respectively. Black triangles indicate microarray probes designed within the transcribed regions. If antisense counterpart on opposite strand has not been overlapped, or not been identified in mice (B and C), microarray probes attempted to detect antisense expression could not be designed. To discover novel conserved NAT, we designed AFAS probes (red triangles) for mouse genes for which the orthologous partner in the human genome has an antisense counterpart.

random-primed target sample, not in the oligo-dT primed target (Figure 4E), indicating that Aard-AS tends to be poly(A)-negative and nuclear-localized.

These data clearly confirm that AFAS probes can detect the expression of antisense transcripts in normal tissues, and that they can also identify transcripts expressed in a tissueand cell-type-specific manner. Detection of such expression dynamics for antisense transcripts is possible only by using the analytical platform targeting the complementary strand of the annotated genes. Thus, AFAS probes, when used within appropriate biological samples and combined with other analytical modalities, can be used to discover genuine functional NATs; this is an advantage over conventional approaches that depend on publicly available cDNA data.

\section{Detection of novel NATs differentially expressed under pathological conditions}

We next checked whether AFAS probes have the ability to detect antisense transcripts in cancerous tissues. Examples of functional antisense transcripts identified in abnormal cells are CDKN2B, WT1, and HBA2 [8,9,29]. These antisense transcripts control the epigenetic status of surrounding genes by DNA methylation or histone modification and thus are thought to affect the expression of their sense partners. To confirm this notion, we applied the AFAS probe technique to the 404 well-characterized genes including oncogenes and tumor suppressors (1752 AFAS probes were successfully designed, giving 4.4 probes per gene on average). We used these probes in microarray experiments based on the GRS/A mouse strain, which frequently suffers from (MMTV)-induced mammary tumors [36].

For the probes designed to detect the sense transcripts, we identified 57 genes showing differential expression. Among these, 48 were up-regulated and 9 were down-regulated within tumor regions, compared with in normal regions, according to a set statistical threshold $(P \leq 0.05$ by Student's $t$-test) (Figure 5 and Additional file 6$)$. Among the up-regulated genes in tumors, 12 genes (Pdcd6 is shown as an example in Additional file 7) showed loss of antisense expression (Figure 5A, right lower), whereas 
A

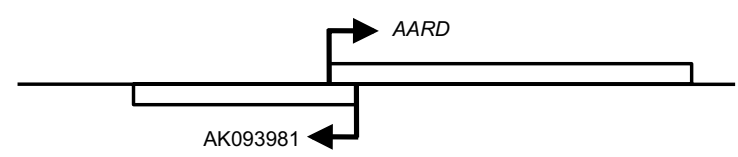

B

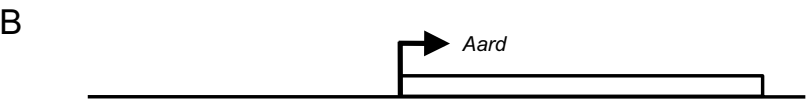

C

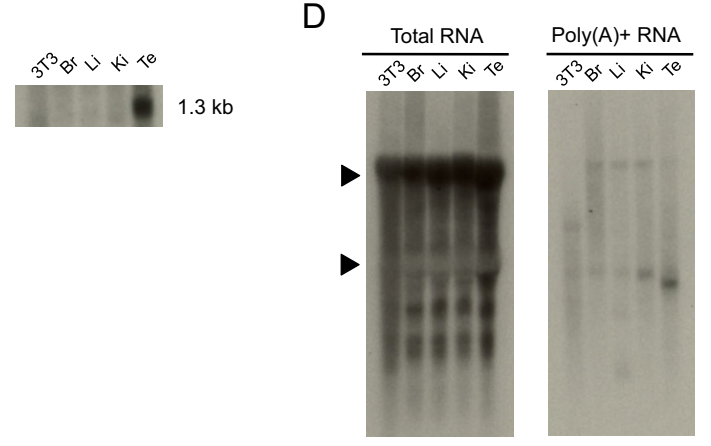

Figure 3

Northern analyses of the sense and antisense transcripts of Aard. (A) Schematic illustration of $A A R D$ and AK093989 (unannotated gene product) in humans. (B) Mouse orthologous partner, Aard. (C) Northern analysis for sense expression of Aard in NIH3T3 cells, brain (Br), liver (Li), kidney (Ki), and testis (Te). (D) Northern analysis for expression of the corresponding antisense transcript for both poly $(\mathrm{A})+$ and total RNA. Triangles indicate 28S (47I0 $\mathrm{nt})$ and I8S (I870 $\mathrm{nt})$ ribosomal RNA.

among the down-regulated genes $\mathrm{Nr} 2 \mathrm{c} 2$ showed up-regulation of its antisense expression in an anti-correlated manner with the sense transcript counterpart (Figure 5A, left upper). These genes are reminiscent of the model in which antisense transcription may lead to the silencing of sense gene expression, such as cyclin-dependent kinase inhibitor $(C D K N 2 B)$ and its antisense counterpart [9]. These genes may be regulated through an antisense-mediated pathway.

Interestingly, the expression of antisense transcripts representing 37 genes (Thbd is shown as an example in Figure 6A) was found to increase, despite the absence of changes in expression of their sense transcript counterparts (Figure 5B). We also identified down-regulated antisense transcripts corresponding to 45 genes (Drd4 is shown as an example in Additional file 7) for which there were no changes in expression of their corresponding sense transcripts. Because ISH using cancerous tissues, like microarray analysis, can detect antisense expression arising from Thbd (thrombomodulin) (Figure 6B-D), there might be more examples of genes for which antisense expression is altered in cancerous tissue but cannot be detected by microarray analysis that targets expression from the sense strand of genes.

\section{Discussion}

This paper shows that microarray probes targeting transcription from the complementary strand of known genes can identify novel NATs, an approach that has not been possible solely on the basis of publicly available cDNA data. Recently described high-density oligonucleotide tiling-array platforms are designed to overview the transcriptional landscape of specific genomic regions at high resolution. By comparison, our platform uses multiple probes to specifically screen for transcription from the antisense strand of known genes. Many previous studies have attempted to identify NATs by DNA microarray analysis using cDNA-oriented custom microarrays or commercially available microarray platforms [37-41]. Since our microarray platform is custom-made and not commercial, it can be applied to any genes or gene loci of interest. Furthermore, our method does not introduce bias from cDNA synthesis between sense and antisense profiling because it does not require specific protocols for target cDNA synthesis for NAT detection. In addition, our microarray platform approach can simultaneously profile sense and antisense expression in one microarray hybridization experiment.

Many NATs detected by AFAS probes were appeared only in the random-primed targets. This was concordance with previous CDNA-based microarray profiling of NAT expression [24]. Whereas poly(A)-plus RNA population is roughly represented by oligo-dT primed cDNAs, whole transcriptome (including the poly(A)-minus RNA population) is represented by cDNAs synthesized by random primers. Therefore, NATs detected by our analysis tend to be poly(A)-negative. Although oligo-dT primers can pick the internal poly(A)-stretches, this is not an issue at the level of microarray-based NAT screening, because the vast majority of the poly(A)-stretch (approximately 90\%) is located within the $3^{\prime}$ end of the transcripts (data not shown).

By designating AFAS probes to human-mouse orthologous genes, we identified many probes showing positive signals. Two of these probes identified transcripts for which in vivo expression was confirmed. Thus, our approach may reveal more, as yet unidentified, conserved NATs; this has not been possible by conventional approaches, as previously reported using cDNA data $[26,31,32]$. Of the individually validated examples (Acaa $1 b$ and Aard), expression of Aard-AS was localized to the nucleus and was detected only in random-primed target samples. In addition, multiple-size hybridized bands pattern was observed especially for total RNA membrane, not for poly(A)+ RNA membrane. This observation is similar to that of previously identified antisense transcripts 
A

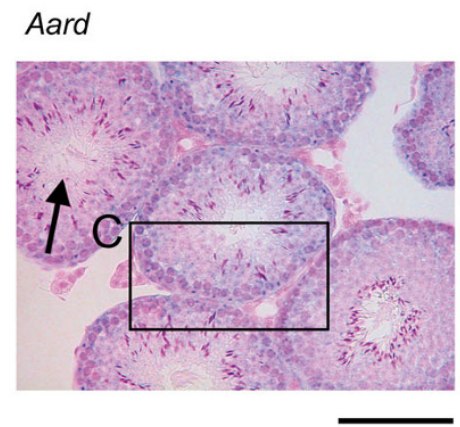

Aard-AS

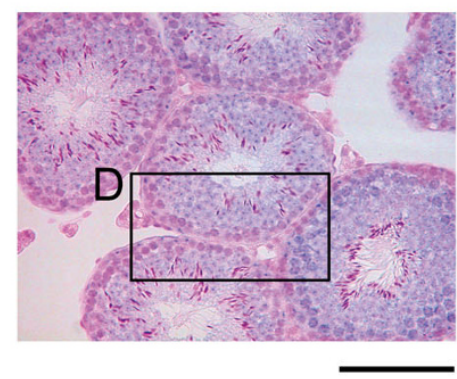

B

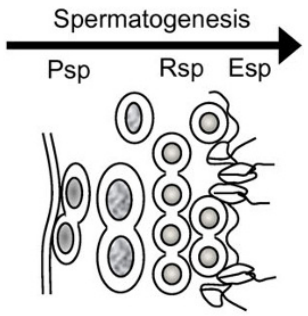

C

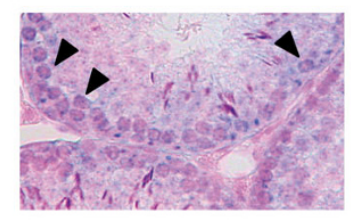

D

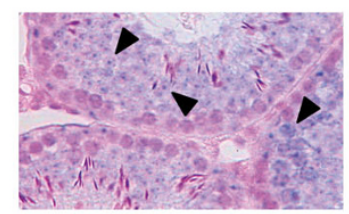

$\mathrm{E}$

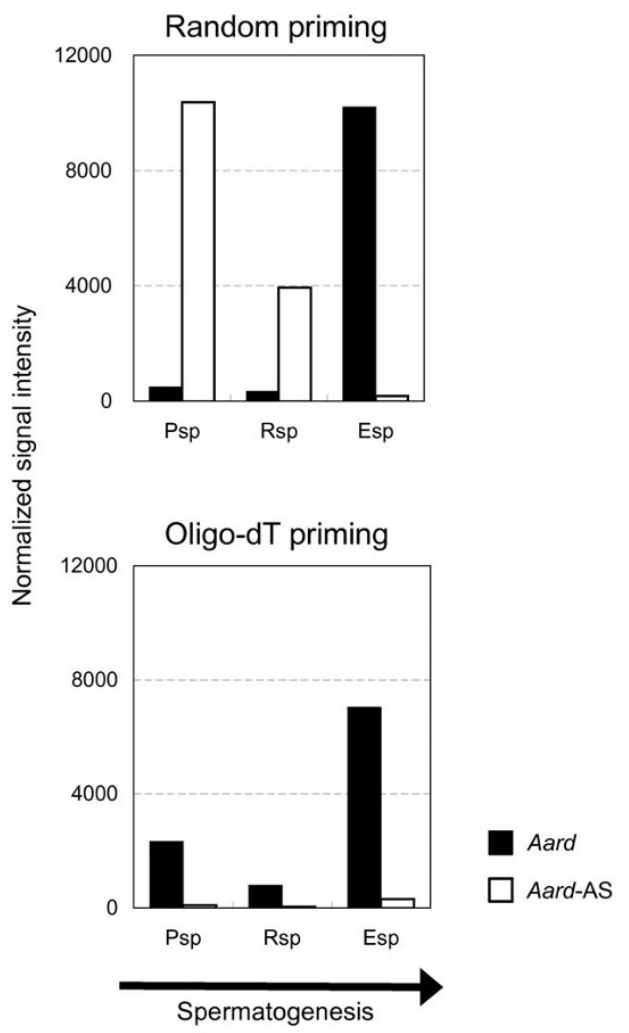

\section{Figure 4}

Expression dynamics of Aard and Aard-AS. (A) In situ hybridization results (seminiferous tubules) for sense (Aard) and antisense (Aard-AS) transcripts. Arrow indicates the direction of spermatogenesis, as illustrated in (B). Scale bars: $100 \mu \mathrm{m}$. (C and D) Enlargement of the corresponding boxes in (A). Arrowheads denote the cytoplasmic signal of Aard and the nuclear signal of Aard-AS. (E) Microarray results of Aard and Aard-AS expression during the spermatogenesis. Black and white bars indicate normalized signal intensity levels of sense and AFAS probe, respectively. Arrow indicates the direction of spermatogenesis (Psp, pachytene spermatocytes; Rsp, round spermatids; Esp, elongated spermatids). Fractionation of germ cells on the basis of the three stages of spermatogenesis in the mouse testis was performed as previously described [53,54].

[24], and this is probably due to heterogeneously sized molecules of Aard-AS transcripts. Because ISH and the microarray data on other antisense transcript examples also show nuclear localization and poly(A)-avoidance (data not shown), it is possible that these features are general characteristics of the antisense transcriptome.

We also designed AFAS probes for well-characterized genes and identified several examples of correlated and anti-correlated expression between the NATs and the corresponding sense transcript within MMTV-induced mammary tumors. We observed differentially expressed genes for which expression of the antisense transcript had changed, whereas that of the sense transcript had not. Given that differential antisense expression might induce changes in epigenetic status, for example in CDKN2B and
CDKN2BAS [9], antisense transcription may cause changes in the methylation status of neighboring genes. This notion can be tested by using methylated DNA immunoprecipitation (MeDIP) and chromatin immunoprecipitation (ChIP) on chip analyses to further characterize the antisense transcriptome and to determine whether specific NATs function as epigenetic regulators. Whereas this study revealed NATs specific to mouse tumors, human clinical samples have also been analyzed to screen for novel NATs by the same methodology; this new study has identified many antisense transcripts showing increased or decreased expression in human colon cancer tissues compared with controls (Saito R., Kohno K., Okada Y., Osada Y., Numata K., Watanabe K., Nakaoka H., Yamamoto N., Kanai A., Yasue H. et al., manuscript in preparation). 
A

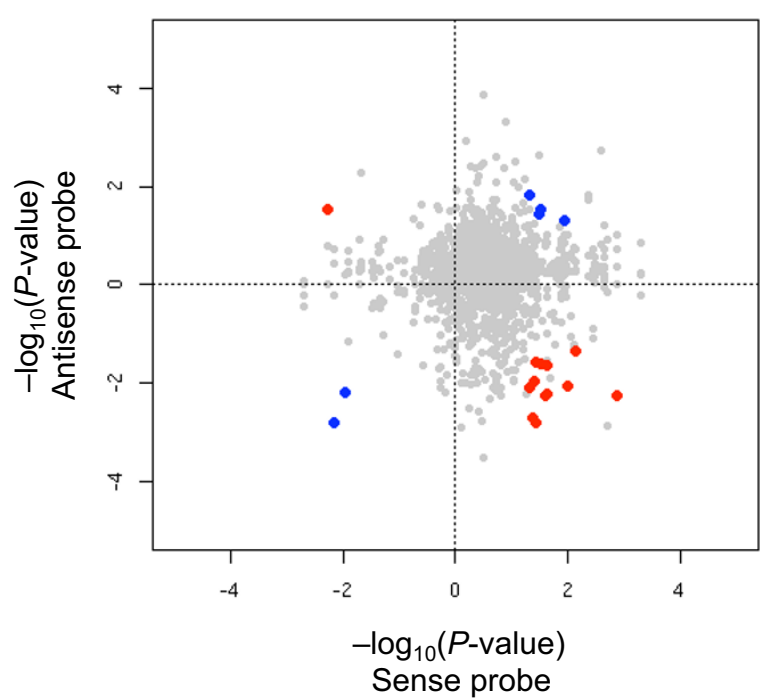

$\mathrm{B}$

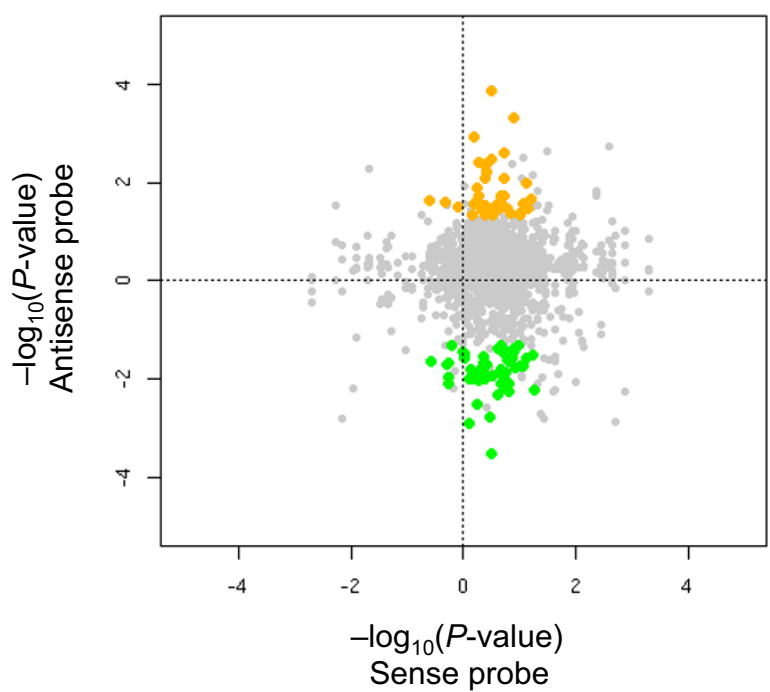

Figure 5

Probes showing differential expression between normal and tumor regions. Differential expression between normal and tumor regions is plotted with log-scaled $P$ value according to Student's $t$-test for sense ( $x$-axis) and antisense ( $y$-axis) expression. In cases where the mean value of signals from normal regions was higher than that of tumors, the value was multiplied by -I. Accordingly, values higher than zero indicate up-regulation, whereas values lower than zero indicate down-regulation, in tumors. (A) Colored points denote significant changes in the expression of both the sense and antisense transcripts in tumors, in a correlated manner (blue) and in an anti-correlated manner (red). The names of the genes indicated by the colored points are listed in additional file 6. (B) Orange and green dots indicate up-regulated and down-regulated antisense expression from genes, respectively, but no apparent changes in sense transcript expression.

Although next-generation high-throughput transcriptome sequencing (RNA-seq) might replace microarray-based expression analyses, antisense transcriptome analysis by sequencing is still under development because of the laborious nature of strand-specific library construction [42]. DNA microarray-based profiling makes it possible to gain a detailed view of specific genes or gene loci and can also provide expression profiles of both poly(A)-plus and poly(A)-minus RNAs.

\section{Conclusion}

We showed here that probes targeting the complementary strand of the annotated genes successfully identify novel NAT expression, including those altered tissue- and tumor-specifically. The results suggest that there are more examples of NATs that cannot not be collected from public cDNA sources. Further functional investigation is required for such dynamically expressed NATs, and the use of microarray platforms targeting both strands of the gene locus will help to narrow down the proper candidates for further functional analyses.

\section{Methods}

\section{Custom microarray construction}

The AFAS probes for detecting NATs were designed to detect antisense transcription originating from genes categorized into three groups: (1) 48 genes in which antisense transcription has been previously reported and 87 imprinted genes in mice, (2) 404 selected well-annotated genes, (3) orthologous genes in NAT loci (detailed definition given below), and (4) randomly selected genes for which there were no cDNA, EST, and CAGE tags in the antisense orientation. For categories (1) and (2), the AFAS probes were designed to correspond to every 500 bases of the antisense strand of the exonic regions of each gene. For category (3), the AFAS probes were designed to correspond to a single specific sequence in each transcript. For category (4), two AFAS probes were designed per transcript. Target region selection for the probe design is summarized in Additional file 8. All probes were computationally designed by using the OligoWiz program [43] and were used in the Agilent $44 \mathrm{~K}$ custom oligoarray platform for single-color microarray analysis. 


\section{A Thrombomodulin $(T h b d)$}
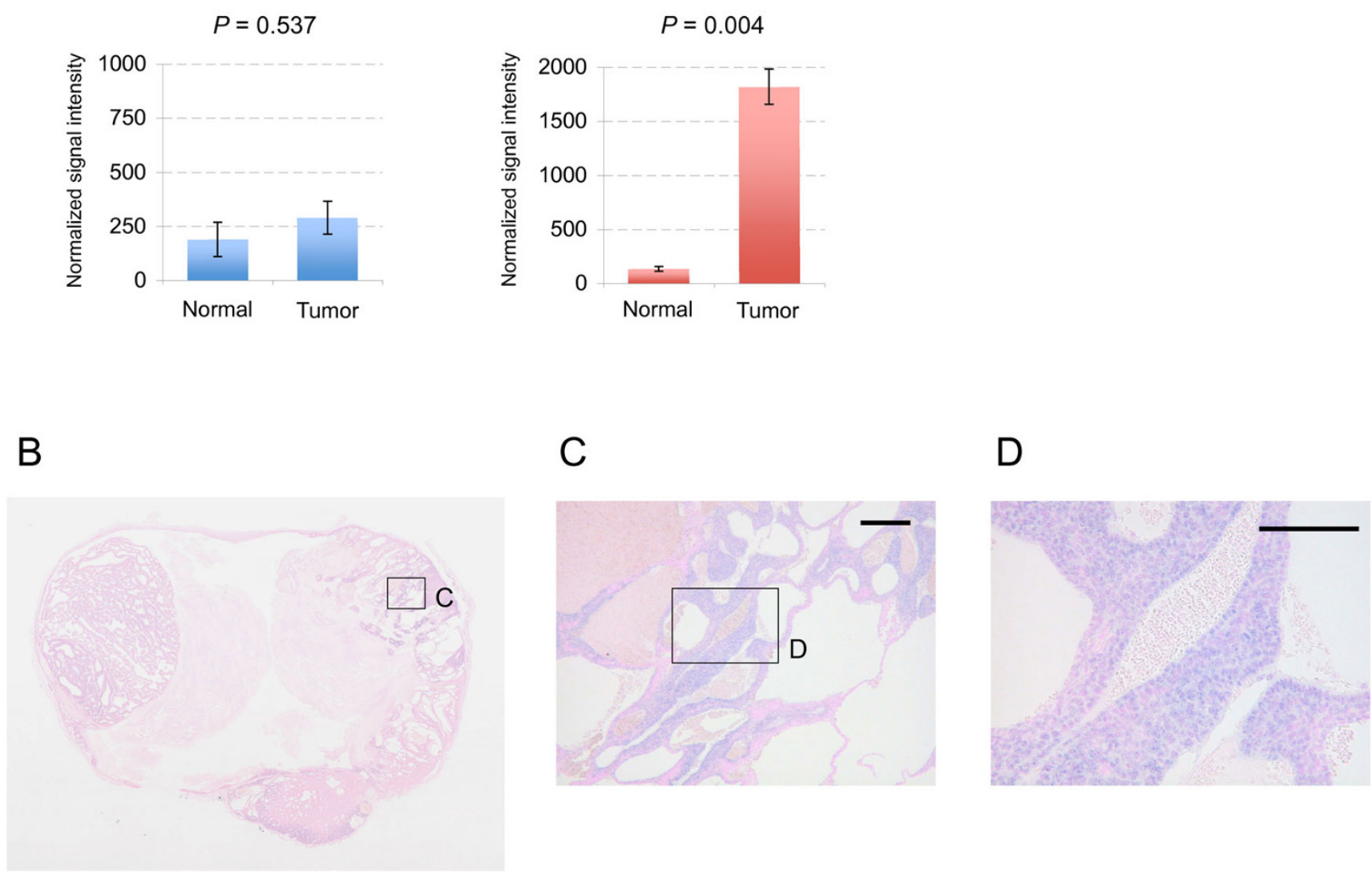

\section{Figure 6}

In situ hybridization of the antisense transcript of Thbd. (A) Microarray results for Thbd, for which expression of the antisense transcript (red bars) has markedly changed in tumor cells but that of the sense transcript (blue bars) has not. (B-D) Results of in situ hybridization of mammary tumor tissue of GRS/A mice for detecting antisense transcription of Thbd. Scale bars: $200 \mu \mathrm{m}(\mathbf{C}) ; 100 \mu \mathrm{m}$ (D).

Target sample preparation for the microarray analysis Total RNA for the mouse (C57BL/6J) microarray experiments was isolated from NIH3T3 cells (fibroblast cell line), SL10 cells (fibroblast cell line), brain, heart, intestine, kidney, liver, lung, placenta (d.p.c. 10.5 and 13.5), spleen, stomach, testis, and thymus. Testis was from C57BL/6J males (8 to 10 weeks), placenta was from pregnant mice, and the other tissue was from both male and female mice. Nuclear and cytoplasmic fractionation of NIH3T3 cells was carried out according to the Protein and RNA Isolation System (PARIS) instructions (Ambion Inc.). For the microarray analysis of murine mammary tumors, RNA samples were collected from normal and cancerous mammary glands of dissected GRS/A mice [36].

\section{Data processing and the accessibility}

Numerical processed signal values (gProcessedSignal) of the Agilent Feature Extraction File were obtained as repre- sentative expression levels for each probe within the array. If a spot had an intensity value lower than five, or if there was no prominent difference between foreground and background signals, then the intensity value was adjusted to five and the corresponding probe was treated as an "absent probe". To perform normalization of signal intensity distribution between multiple arrays, the whole mean signal of every hybridization experiment was adjusted to that of the data from SL10 cells by oligo-dT priming. Probes with intensity values lower than five, as well as being flagged as "saturated", were discarded for the interarray-normalization step. Tissue-specificity of the expression signals was evaluated according to $\tau$ measurement [44]. The raw data from the microarray analyses were deposited in the NCBI Gene Expression Omnibus (GEO) under accession number GSE14568 [45]. Expression data as well as a simplified genomic structure can be accessed via an originally constructed viewer [46]. 


\section{In silico identification of orthologous genes in NAT loci} To identify orthologous genes in NAT loci (Figure 2), we initially performed in silico identification of sense-antisense pairs by the same procedures as previously published [47], by using the latest full-length cDNA collections [33,48], NCBI RefSeq mRNA [49] and the UniGene collection [50]. This identified 3524 and 5351 exonoverlapping sense-antisense pairs in humans and mice, respectively. Genomic synteny data between human and mouse (defined by BLASTZ derived from UCSC [51]) was then exploited to determine whether each identified pair was located within the syntenic region between the two species. Those pairs located within the syntenic regions were retained for the orthologous relationship validation. The orthologous relationship between the genes located within the syntenic regions was defined according to the orthologous gene table from the BioMart Project [52]. Finally, 648 genes are identified as orthologous genes for which NAT was identified in human cDNAs but not in mouse cDNAs. AFAS probes for these (635 of 648) were successfully designed.

\section{Northern hybridization analyses}

RNA from mouse tissues (C57BL/6J, 8 to 10 weeks, male and female mixed), and the NIH3T3 was isolated by using Trizol reagent (Invitrogen Corporation). Northern analyses were performed as previously described [24]. Loading of equal amounts of RNA samples was confirmed by visualization of ethidium bromide-stained RNA in the gel. Probes specific for sense and antisense of Acaa1b (NM_146230), Aard (NM_175503), and Thbd (NM_009 378) were amplified by the PCR (see Additional file 9). All the probe sequences contained their corresponding microarray probe sequences. cDNA fragments were cloned to the pGEM-T Easy Vector (Promega Corporation), and strand-specific cRNA was prepared for hybridization.

\section{In situ hybridization}

Probes specific for sense and antisense of Acaa1b (NM_146230), Aard (NM_175503), and Thbd (NM_009378) were amplified by the PCR (see Additional file 9). All the probe sequences contained their corresponding microarray probe sequences. The amplified fragment was sub-cloned into pGEMT-Easy vector (Promega) and was used for generation of sense or antisense RNA probes. Paraffin-embedded testis sections (6 $\mu \mathrm{m})$ of normal adult mouse (C57BL/ 6 mouse, male, 8 weeks) were obtained from Genostaff Co., Ltd. For in situ hybridization the sections were hybridized with digoxigenin-labeled RNA probes at $60^{\circ} \mathrm{C}$ for $16 \mathrm{~h}$. The bound label was detected using NBT-BCIP, an alkaline phosphate color substrate. The sections were counterstained with Kernechtrot (Muto Pure Chemicals Co., Ltd.). Probe sequence of negative control experiment was selected from Oryza sativa putative leaf protein (NM_197207) (see Additional file 5 and 10).

\section{Real-time quantitative $R$ T-PCR}

cDNA was initially synthesized with gene-specific reverse primers (Acaa1b-AS and Gapdh) from selected tissue RNA (Brain, Testis, Kidney, and Liver), then subjected to quantitative RT-PCR. Gene expression level was normalized with Gapdh. Primers are listed in Additional file 11.

\section{Authors' contributions}

$\mathrm{KN}$ wrote the manuscript, with editing by RS, AK, KA, and HK Microarray design and bioinformatics analyses were performed by KN, YOs, and YOk GRS/A mice were prepared and dissected by NH. HN and NY developed the original viewer for the expression data. KW performed the microarray experiments, and KO performed the in-situ hybridization experiments. CK performed quantitative RT-PCR analysis. HK organized and directed the project.

\section{Additional material}

\author{
Additional file 1 \\ Signal intensities from AFAS probes for Tsix. AFAS probes designed for \\ Tsix, which reflects the abundance of Xist RNA, detected expression in the \\ 11 adult mouse tissues (mixed males and females), but not in the testis. \\ Click here for file \\ [http://www.biomedcentral.com/content/supplementary/1471- \\ 2164-10-392-S1.pdf]
}

\section{Additional file 2}

Antisense expression of mouse imprinted genes. AFAS probes for several imprinted genes (Igf2r, Kcnq1, Gnas, Dio3, and Ube3a), which are known to give rise to antisense transcripts, gave prominent signals. Click here for file

[http://www.biomedcentral.com/content/supplementary/14712164-10-392-S2.pdf]

\section{Additional file 3}

Numbers of valid probes in adult mouse tissue profiling. A significantly higher number of AFAS probes than sense probes detected transcripts only within random-primed samples, but not among the oligo-dT primed targets.

Click here for file

[http://www.biomedcentral.com/content/supplementary/14712164-10-392-S3.pdf]

\section{Additional file 4}

Highest signal intensities from expression profiling of the 12 normal adult tissues. Probes of 120 genes gave signals with a higher than average intensity according to inter-array normalization.

Click here for file

[http://www.biomedcentral.com/content/supplementary/14712164-10-392-S4.pdf]

\section{Additional file 5}

Expression analyses of sense and antisense transcripts of Acaa1b. Quantitative RT-PCR, ISH and microarray analyses were able to detect this transcript within the testis and kidney.

Click here for file

[http://www.biomedcentral.com/content/supplementary/14712164-10-392-S5.pdf] 


\section{Additional file 6 \\ List of genes for which expression of the antisense transcript and sense transcript markedly changed in tumors. Forty-eight were up-regulated and nine were down-regulated within tumor regions, compared with in normal regions. \\ Click here for file \\ [http://www.biomedcentral.com/content/supplementary/1471- 2164-10-392-S6.pdf]}

\section{Additional file 7}

Changes in expression of Pdcd6 and Drd4.

Click here for file

[http://www.biomedcentral.com/content/supplementary/14712164-10-392-S7.pdf]

\section{Additional file 8}

Selection of target regions for microarray probe design. Target region selection for the probe design is summarized.

Click here for file

[http://www.biomedcentral.com/content/supplementary/1471-

2164-10-392-S8.pdf]

\section{Additional file 9}

List of primers for PCR amplification of cDNA fragments to generate probes for Northern blot analysis and in situ hybridization. Primers to amplify probes specific for sense and antisense of Acaa1b, Aard, and Thbd are listed.

Click here for file

[http://www.biomedcentral.com/content/supplementary/14712164-10-392-S9.pdf]

\section{Additional file 10}

Negative control experiment of Aard in situ hybridization.

Click here for file

[http://www.biomedcentral.com/content/supplementary/1471-

2164-10-392-S10.pdf]

\section{Additional file 11}

Primers for real-time quantitative RT-PCR. Primers for real-time quantitative RT-PCR (Gapdh and Acaa1b-AS) are listed.

Click here for file

[http://www.biomedcentral.com/content/supplementary/1471-

2164-10-392-S11.pdf]

\section{Acknowledgements}

The authors acknowledge Yutaka Watanabe and Takahiro Doi (RIKEN BRC) for their helpful discussions, Hidemasa Kato (Saitama Medical University) for critical reading of the manuscript, Shinichi Kashiwabara (Tsukuba University) for fractionation of the testis germ cells, and Toutai Mitsuyama (Computational Biology Research Center, Advanced Industrial Science and Technology, Japan) and Kiyoshi Asai (Tokyo University) for bioinformatic support. We also thank Naoto Kaneko (Tsukuba University), Kouichi Tatsuguchi and Yukiaki Kikuta (C's Lab Co. Ltd), members of Genostaff Inc., and staff at Hokkaido System Science Co. Ltd for technical and experimental support. This work was supported in part by grants from the Non-coding RNA Project by the New Energy and Industrial Technology Development Organization (NEDO) of Japan; and by a Research Fellowship of the Japan Society for the Promotion of Science (JSPS) for Young Scientists to K.N.

\section{References}

I. Lapidot M, Pilpel Y: Genome-wide natural antisense transcription: coupling its regulation to its different regulatory mechanisms. EMBO Rep 2006, 7(1 2): I 1 16-1222.

2. Lavorgna G, Dahary D, Lehner B, Sorek R, Sanderson CM, Casari G: In search of antisense. Trends Biochem Sci 2004, 29(2):88-94.

3. Shibata S, Lee JT: Tsix transcription- versus RNA-based mechanisms in Xist repression and epigenetic choice. Curr Biol 2004, I 4(19): 1747-I754.

4. Sleutels F, Zwart R, Barlow DP: The non-coding Air RNA is required for silencing autosomal imprinted genes. Nature 2002, 41 5(6873):810-8I3.

5. O'Neill MJ: The influence of non-coding RNAs on allele-specific gene expression in mammals. Hum Mol Genet 2005, I4(Spec No I):R I I3-I20.

6. Pandey RR, Mondal T, Mohammad F, Enroth S, Redrup L, Komorowski J, Nagano T, Mancini-Dinardo D, Kanduri C: Kcnq l ot I antisense noncoding RNA mediates lineage-specific transcriptional silencing through chromatin-level regulation. $\mathrm{Mol}$ Cell 2008, 32(2):232-246.

7. Babak T, Deveale B, Armour C, Raymond C, Cleary MA, Kooy D van der, Johnson JM, Lim LP: Global survey of genomic imprinting by transcriptome sequencing. Curr Biol 2008, I 8(22): I735-I74I.

8. Tufarelli C, Stanley JA, Garrick D, Sharpe JA, Ayyub H, Wood WG, Higgs DR: Transcription of antisense RNA leading to gene silencing and methylation as a novel cause of human genetic disease. Nat Genet 2003, 34(2): 157-165.

9. Yu W, Gius D, Onyango P, Muldoon-Jacobs K, Karp J, Feinberg AP, Cui $\mathrm{H}$ : Epigenetic silencing of tumour suppressor gene p I5 by its antisense RNA. Nature 2008, 45 I (7 I 75):202-206.

10. Borsani O, Zhu J, Verslues PE, Sunkar R, Zhu JK: Endogenous siRNAs derived from a pair of natural cis- antisense transcripts regulate salt tolerance in Arabidopsis. Cell 2005, I23(7): |279-129|.

II. Jin H, Vacic V, Girke T, Lonardi S, Zhu JK: Small RNAs and the regulation of cis-natural antisense transcripts in Arabidopsis. BMC Mol Biol 2008, 9:6.

12. Katiyar-Agarwal S, Gao S, Vivian-Smith A, Jin $\mathrm{H}$ : A novel class of bacteria-induced small RNAs in Arabidopsis. Genes Dev 2007, 2 I(23):3 | 23-3 | 34.

13. Katiyar-Agarwal S, Morgan R, Dahlbeck D, Borsani O, Villegas A Jr, Zhu JK, Staskawicz BJ, Jin H: A pathogen-inducible endogenous siRNA in plant immunity. Proc Natl Acad Sci USA 2006, I03(47): I8002-18007.

14. Lu C, Jeong DH, Kulkarni K, Pillay M, Nobuta K, German R, Thatcher SR, Maher C, Zhang L, Ware D, et al:: Genome-wide analysis for discovery of rice microRNAs reveals natural antisense microRNAs (nat-miRNAs). Proc Natl Acad Sci USA 2008, I 05( I 2):4951-4956.

15. Zhou X, Sunkar R, Jin H, Zhu JK, Zhang W: Genome-wide identification and analysis of small RNAs originated from natural antisense transcripts in Oryza sativa. Genome Res 2009, I9(I):70-78.

16. Carlile M, Swan D, Jackson K, Preston-Fayers K, Ballester B, Flicek P, Werner A: Strand selective generation of endo-siRNAs from the $\mathrm{Na}$ /phosphate transporter gene Slc34al in murine tissues. Nucleic Acids Res 2009, 37(7):2274-2282.

17. Okamura K, Balla S, Martin R, Liu N, Lai EC: Two distinctmechanisms generate endogenous siRNAs from bidirectional transcription in Drosophila melanogaster. Nat Struct Mol Biol 2008, I 5(6):58|-590.

18. Watanabe T, Totoki Y, Toyoda A, Kaneda M, Kuramochi-Miyagawa S, Obata Y, Chiba H, Kohara Y, Kono T, Nakano T, et al.: Endogenous siRNAs from naturally formed dsRNAs regulate transcripts in mouse oocytes. Nature 2008, 453:539-543.

19. Zhang Y, Liu XS, Liu QR, Wei L: Genome-wide in silico identification and analysis of cis natural antisense transcripts (cisNATs) in ten species. Nucleic Acids Res 2006, 34( I 2):3465-3475.

20. Cheng J, Kapranov P, Drenkow J, Dike S, Brubaker S, Patel S, Long J, Stern D, Tammana H, Helt G, et al.: Transcriptional maps of I0 human chromosomes at 5-nucleotide resolution. Science 2005, 308(5725): I |49-I| 54.

21. Steinhoff C, Vingron M: Normalization and quantification of differential expression in gene expression microarrays. Brief Bioinform 2006, 7(2): 166-177. 
22. Nepveu A, Marcu KB: Intragenic pausing and anti-sense transcription within the murine c-myc locus. EMBO J 1986, 5( I I ):2859-2865.

23. Coker RK, Laurent GJ, Dabbagh K, Dawson J, McAnulty RJ: A novel transforming growth factor beta2 antisense transcript in mammalian lung. Biochem J 1998, 332(Pt 2):297-30I.

24. Kiyosawa H, Mise N, Iwase S, Hayashizaki $\mathrm{Y}$, Abe K: Disclosing hidden transcripts: mouse natural sense-antisense transcripts tend to be poly(A) negative and nuclear localized. Genome Res 2005, I 5(4):463-474.

25. David L, Huber W, Granovskaia M, Toedling J, Palm CJ, Bofkin L, Jones T, Davis RW, Steinmetz LM: A high-resolution map of transcription in the yeast genome. Proc Natl Acad Sci USA 2006, I 03( I 4):5320-5325.

26. Numata K, Okada Y, Saito R, Kiyosawa H, Kanai A, Tomita M: Comparative analysis of cis- encoded antisense RNAs in eukaryotes. Gene 2007, 392(I-2):|34-|4|.

27. Sun M, Hurst LD, Carmichael GG, Chen J: Evidence for a preferential targeting of $3^{\prime}$-UTRs by cis- encoded natural antisense transcripts. Nucleic Acids Res 2005, 33(I 7):5533-5543.

28. Imamura T, Yamamoto S, Ohgane J, Hattori N, Tanaka S, Shiota K: Non-coding RNA directed DNA demethylation of SphkI CpG island. Biochem Biophys Res Commun 2004, 322(2):593-600.

29. Dallosso AR, Hancock AL, Malik S, Salpekar A, King-Underwood L, Pritchard-Jones K, Peters J, Moorwood K, Ward A, Malik KT, et al.: Alternately spliced WTI antisense transcripts interact with WTI sense RNA and show epigenetic and splicing defects in cancer. RNA 2007, I 3( I 2):2287-2299.

30. Ohhata T, Hoki Y, Sasaki H, Sado T: Crucial role ofantisense transcription across the Xist promoter in Tsix-mediated Xist chromatin modification. Development 2008, I35(2):227-235.

31. Engstrom PG, Suzuki H, Ninomiya N, Akalin A, Sessa L, Lavorgna G, Brozzi A, Luzi L, Tan SL, Yang L, et al.: Complex loci in human and mouse genomes. PLoS Genet 2006, 2(4):e47.

32. Galante PA, Vidal DO, de Souza JE, Camargo AA, de Souza SJ: Senseantisense pairs in mammals: functional and evolutionary considerations. Genome Biol 2007, 8(3):R40.

33. Carninci P, Kasukawa T, Katayama S, Gough J, Frith MC, Maeda N, Oyama R, Ravasi T, Lenhard B, Wells $C$, et al: The transcriptional landscape of the mammalian genome. Science 2005 309(5740): I559-1563.

34. Birney E, Stamatoyannopoulos JA, Dutta A, Guigo R, Gingeras TR Margulies EH, Weng Z, Snyder M, Dermitzakis ET, Thurman RE, et al.: Identification and analysis of functional elements in $1 \%$ of the human genome by the ENCODE pilot project. Nature 2007 447(7 | 46):799-8I6

35. Svingen T, Beverdam A, Verma P, Wilhelm D, Koopman P: Aard is specifically up-regulated in Sertoli cells during mouse testis differentiation. Int J Dev Biol 2007, 5 I (3):255-258.

36. van Nie R, Hilgers J: Genetic analysis of mammary tumor induction and expression of mammary tumor virus antigen in hormone-treated ovariectomized GR mice. J Natl Cancer Inst 1976, 56(I):27-32.

37. Werner A, Schmutzler G, Carlile M, Miles CG, Peters H: Expression profiling of antisense transcripts on DNA arrays. Physiol Genomics 2007, 28(3):294-300.

38. Oeder S, Mages J, Flicek P, Lang R: Uncovering information on expression of natural antisense transcripts in Affymetrix MOE430 datasets. BMC Genomics 2007, 8:200

39. Vallon-Christersson J, Staaf J, Kvist A, Medstrand P, Borg A, Rovira C: Non-coding antisense transcription detected by conventional and single-stranded cDNA microarray. BMC Genomics 2007, 8:295.

40. Gyorffy A, Surowiak P, Tulassay Z, Gyorffy B: Highly expressed genes are associated with inverse antisense transcription in mouse. J Genet 2007, 86(2): 103-109.

41. Ge X, Rubinstein WS, Jung YC, Wu Q: Genome-wide analysis of antisense transcription with Affymetrix exon array. $B M C$ Genomics 2008, 9:27

42. Wang Z, Gerstein M, Snyder M: RNA-Seq: a revolutionary tool for transcriptomics. Nat Rev Genet 2009, I O(I):57-63.

43. Wernersson $\mathrm{R}$, Nielsen $\mathrm{HB}$ : OligoWiz 2.0-integrating sequence feature annotation into the design of microarray probes. Nucleic Acids Res 2005:W6II-6I5.

44. Yanai I, Benjamin H, Shmoish M, Chalifa-Caspi V, Shklar M, Ophir R, Bar-Even A, Horn-Saban S, Safran M, Domany E, et al.: Genome- wide midrange transcription profiles reveal expression level relationships in human tissue specification. Bioinformatics 2005, 2 I (5):650-659.

45. National Center for Biotechnology Information Gene Expression Omnibus [http://www.ncbi.nlm.nih.gov/geo/]

46. Antisense Viewer [http://www.brc.riken.jp/archives/Kiyosawa/ BMC Genomics09/]

47. Okada Y, Tashiro C, Numata K, Watanabe K, Nakaoka H, Yamamoto N, Okubo K, Ikeda R, Saito R, Kanai A, et al.: Comparative expression analysis uncovers novel features of endogenous antisense transcription. Hum Mol Genet 2008, I 7( I I): I63 I-I640.

48. Ota T, Suzuki Y, Nishikawa T, Otsuki T, Sugiyama T, Irie R, Wakamatsu A, Hayashi K, Sato H, Nagai K, et al.: Complete sequencing and characterization of 21,243 full-length human cDNAs. Nat Genet 2004, 36:40-45.

49. Pruitt KD, Tatusova T, Maglott DR: NCBI reference sequences (RefSeq): a curated non-redundant sequence database of genomes, transcripts and proteins. Nucleic Acids Res 2007:D6 I-65.

50. Wheeler DL, Church DM, Federhen S, Lash AE, Madden TL, Pontius JU, Schuler GD, Schriml LM, Sequeira E, Tatusova TA, et al:: Database resources of the National Center for Biotechnology. Nucleic Acids Res 2003, 3 I (I):28-33.

51. Schwartz S, Kent WJ, Smit A, Zhang Z, Baertsch R, Hardison RC, Haussler D, Miller W: Human-mouse alignments with BLASTZ. Genome Res 2003, I3(I): I03-107.

52. BioMart Project [http://www.biomart.org/]

53. Bellve AR, Cavicchia JC, Millette CF, O'Brien DA, Bhatnagar YM, Dym $M$ : Spermatogenic cells of the prepuberal mouse. Isolation and morphological characterization. J Cell Biol I977, 74(I):68-85.

54. Romrell LJ, Bellve AR, Fawcett DW: Separation of mouse spermatogenic cells by sedimentation velocity. A morphological characterization. Dev Biol I976, 49(I): II9-I3।.
Publish with Biomed Central and every scientist can read your work free of charge

"BioMed Central will be the most significant development for disseminating the results of biomedical research in our lifetime. "

Sir Paul Nurse, Cancer Research UK

Your research papers will be:

- available free of charge to the entire biomedical community

- peer reviewed and published immediately upon acceptance

- cited in PubMed and archived on PubMed Central

- yours - you keep the copyright

Submit your manuscript here:

http://www.biomedcentral.com/info/publishing_adv.asp
BiolMedcentral 